\title{
ENSAIO DE PROFICIÊNCIA PARA ANÁLISE DE DITIOCARBAMATOS EM POLPA DE BANANA
}

\author{
Lucia Helena Pinto Bastos*, Heloisa Cronemberger de Araújo Góes, Maria Helena Wohlers Morelli Cardoso, Adherlene \\ Vieira Gouvêa, Denise de Paula Dias, Renato Rubim Ribeiro de Almeida, Armi Nóbrega e Shirley Abrantes \\ Instituto Nacional de Controle de Qualidade em Saúde, Fundação Oswaldo Cruz, Av. Brasil 4365, \\ 21040-900, Rio de Janeiro - RJ, Brasil
}

Recebido em 8/9/05; aceito em 7/4/06; publicado na web em 11/8/06

\begin{abstract}
PROFICIENCY ASSAY OF THE DITHIOCARBAMATES IN BANANA PULP ANALYSIS. A proficiency assay of the determination of dithiocarbamate pesticide residues in banana was carried out. Fourteen laboratories participated in this study. Homogeneity and stability testing were performed by INCQS on the samples sent to the laboratories. Analytical results supplied by the pesticide residues laboratory of the VWA/KvW, Amsterdam, Holland, were used to define the designated value for the thiram concentration in the study samples. Results: Fifty percent of the participating laboratories had satisfactory results. Efforts are needed to improve the precision of the analytical results and to decrease the number of false negative results observed.
\end{abstract}

Keywords: pesticides; dithiocarbamates; interlaboratorial.

\section{INTRODUÇÃO}

O Brasil, como um país de extensa área rural e clima favorável, é um dos maiores produtores e exportadores de produtos agrícolas do mundo. Entretanto, muitos agricultores ainda não seguem as boas práticas agrícolas e, muitas vezes, utilizam agrotóxicos indiscriminadamente, tanto no que se refere à quantidade como à qualidade desses produtos. Tal fato torna-se uma preocupação para a saúde pública, devido aos danos causados ao trabalhador rural e ao consumidor.

Os ditiocarbamatos (DTCs) pertencem a um grupo de agrotóxicos organossulfurados empregados na agricultura com ação fungicida. No Brasil existem registrados seis tipos de substâncias da classe dos ditiocarbamatos como ingredientes ativos, para quarenta e um diferentes tipos de cultura ${ }^{1}$.

A relevância toxicológica dessas substâncias deve-se a seus dois maiores produtos de degradação, etileno tiouréia (ETU) e propilenotiouréia (PTU), suspeitos de serem bociogênicos, carcinogênicos e mutagênicos em $\operatorname{ratos}^{2,3}$. No Brasil, o uso de ditiocarbamatos em culturas é intenso, conforme relatado pelo programa gerenciado pela Agência Nacional de Vigilância Sanitária (ANVISA), o Programa Nacional de Análise de Resíduos de Agrotóxicos em Alimentos (PARA) 4

O monitoramento de agrotóxicos em alimentos é importante para avaliar os níveis das frutas e verduras produzidas e comercializadas no país; verificar se os limites máximos de resíduos permitidos (LMR) estabelecidos pela legislação brasileira estão sendo de fato respeitados; verificar se agrotóxicos não registrados na ANVISA estão sendo utilizados ilegalmente no país; investigar se agrotóxicos devidamente registrados na ANVISA estão sendo utilizados de forma inadequada e, obter dados mais confiáveis para a estimativa da ingestão diária de resíduos de agrotóxicos pela população, uma informação fundamental ao realizar o registro de novos agrotóxicos e/ ou renovar o registro dos já autorizados ${ }^{5}$.

Poucos são os laboratórios que realizam ensaio de determinação de resíduos de agrotóxicos em alimentos no Brasil. Os objeti-

*e-mail: lucia.bastos@incqs.fiocruz.br vos das análises realizadas por esses laboratórios normalmente são controle da qualidade de produção, pesquisa, exportação e proteção da saúde pública. Os laboratórios da rede pública trabalham majoritariamente com os últimos dois objetivos.

Para prover confiabilidade aos resultados obtidos nos complexos ensaios de análise de agrotóxicos é importante a implantação, pelos laboratórios, do sistema da qualidade baseado na Norma NBR ISO/IEC $17025^{6}$.

$\mathrm{O}$ ensaio de proficiência (EP) é uma ferramenta prevista nesta Norma que deve ser utilizado pelos laboratórios de ensaio para verificação da qualidade dos resultados por ele produzidos. Com a crescente demanda por provas regulares e independentes de competência pelos organismos regulatórios e clientes, os laboratórios vêm, cada vez mais, participando destes ensaios.

A oferta internacional de EP é boa, entretanto, os custos cobrados para a participação são, normalmente, muito elevados, o que, em alguns casos, inviabiliza a participação do laboratório em um número maior de ensaios. Além disso, as dificuldades encontradas na importação das amostras acarreta, muitas vezes, na chegada de materiais fora do prazo e em condições inadequadas de temperatura e integridade.

\section{PARTE EXPERIMENTAL}

\section{Preparo das amostras}

As bananas foram coletadas na cidade do Rio de Janeiro, em local de plantação orgânica (sem aplicação de agrotóxicos). Foi realizada análise prévia da cultura, confirmando-se, assim, a ausência de agrotóxicos. Após a remoção da casca, as bananas foram trituradas em liqüidificador e a polpa foi transferida para recipientes de plástico, que ficaram armazenados a $-18{ }^{\circ} \mathrm{C}$ por 3 dias, antes de seu envio aos laboratórios participantes do EP. Ao todo, foram envasados 90 frascos contendo a polpa de banana.

Uma solução do agrotóxico tiram (Tiram, Dr. Ehrenstorfer, Alemanha) em acetona (Omnisolv, Merk, Brasil) com concentração $54,60 \mu \mathrm{g} / \mathrm{mL}$ foi preparada, seguindo as Boas Práticas de Laboratório $(\mathrm{BPL})^{7}$.

Dentre os procedimentos da BPL cabe ressaltar a especificação, 
o preparo e o armazenamento dos materiais de referência (MR). São requisitos da especificação possuir alta pureza e ser rastreável através de certificados de análise reconhecidos por órgãos certificadores ${ }^{7}$.

As soluções dos MR preparadas devem ser claramente identificadas e permitirem total rastreabilidade dos materiais de referência. Devem ser acondicionadas em frascos âmbar com septo de teflon e guardadas em refrigeradores à temperatura de $-18{ }^{\circ} \mathrm{C}$, com controle de temperatura.

Foram preparados $100 \mathrm{~mL}$ da solução e este conteúdo foi redistribuído em porções de 1,0 mL em ampolas de vidro de cor âmbar. A solução teve sua estabilidade testada por 6 meses, não demonstrando degradação nesse período.

\section{Envio das amostras}

Dezessete laboratórios inscreveram-se para participação no EP em agrotóxicos organizado pelo INCQS e quatorze enviaram os resultados. O ensaio foi codificado como EP/INCQS AG4.

A cada laboratório participante foram enviadas 4 amostras de $200 \mathrm{~g}$ da polpa de banana, envasadas e congeladas, e 3 frascos contendo $1 \mathrm{~mL}$ da solução de tiram para fortificação. As amostras de banana foram identificadas como "Branco", "Amostra 1", "Amostra 2" e "Amostra 3", e os frascos de tiram, como "Solução para Amostra 1", "Solução para Amostra 2" e "Solução para Amostra 3", respectivamente.

Uma das amostras foi utilizada como "branco" e as outras 3 foram analisadas seguindo instruções enviadas. Resumidamente, foi solicitada a transferência de $200 \mathrm{~g}$ da polpa de banana de uma das amostras para o sistema de reação e, em seguida, quantitativamente, a transferência do conteúdo do frasco contendo a solução de tiram da amostra correspondente. Imediatamente após esta operação, orientou-se que se iniciasse o procedimento analítico.

Todos os procedimentos de recebimento da amostra, informações sobre metodologias analíticas e envio de resultados foram feitos em formulários específicos, enviados a cada participante.

\section{Teste de homogeneidade}

Dentre as 80 amostras de polpa de banana inicialmente envasadas, 10 foram aleatoriamente separadas e analisadas no INCQS em duplicata. Na análise foram seguidas as mesmas instruções enviadas aos laboratórios participantes. O teste de homogeneidade foi realizado segundo o recomendado no "The International Harmonised Protocol for the Proficiency Testing of Analytical Chemistry Laboratories" da IUPAC ${ }^{8}$. Para tal, após a determinação quantitativa do analito, utilizou-se uma análise de variância fator único ("one-way" ANOVA) para estimar a variância amostral (entre amostras) e analítica (intra-amostras - duplicatas) e comparouse a estimativa do desvio padrão amostral com o desvio padrão do método analítico utilizado9.

\section{Teste de estabilidade}

O teste de estabilidade visa identificar se há reprodutibilidade nas determinações dos agrotóxicos ao longo do tempo. A avaliação foi realizada utilizando-se a análise de resíduos da regressão linear.

Para o teste de estabilidade foram analisadas outras 10 amostras de polpa de banana, também separadas aleatoriamente dentre as 80 preparadas inicialmente. Estas foram armazenadas em congelador $\left(-18^{\circ} \mathrm{C}\right)$, descongeladas e analisadas, nos tempos $0,7,14,21$ e 28 dias, isto é, contando-se desde o momento do envio das amostras aos laboratórios participantes até a data limite estipulada para recebimento dos resultados.

\section{Obtenção do valor designado}

O valor designado no presente EP foi o resultado emitido pelo laboratório "Pesticide Analysis Group of the Dutch Food and Consumer Product Safety Authority" (VWA/KvW) - Amsterdã/ Holanda, que é acreditado pelo "Dutch Accreditation Council" (RvA). Foram enviadas 3 amostras para esse laboratório, e cada uma foi analisada em triplicata. A média dos resultados apresentados por esse laboratório foi o valor designado.

\section{Metodologias analíticas utilizadas}

A metodologia atualmente utilizada para determinação de ditiocarbamatos baseia-se na detecção fotométrica do dissulfeto de carbono $\left(\mathrm{CS}_{2}\right)$, liberado na hidrólise ácida dos DTCs contidos nos alimentos. O complexo cúprico formado é determinado espectrofotometricamente ${ }^{10,11}$.

Como metodologias alternativas ao método espectrofotométrico clássico, surgiram as metodologias de análise cromatográfica do $\mathrm{CS}_{2}$ liberado em isoctano ${ }^{12,13}$. A detecção cromatográfica pode ser por captura de elétrons, fotometria em chama ou detecção seletiva por massa.

Recentemente demonstrou-se ser a metodologia tradicional, a espectrofotométrica, compatível com a cromatográfica. Entretanto, o método cromatográfico apresenta maior sensibilidade, rapidez e utiliza menor quantidade de solventes ${ }^{14}$.

Apesar da legislação especificar diferentes produtos ditiocarbamatos para diferentes culturas, esta contempla o limite máximo residual em $\mathrm{CS}_{2}$ para todos, não distinguindo os diferentes ditiocarbamatos utilizados ${ }^{1}$.

\section{Análise estatística dos resultados enviados pelos participantes}

A fim de avaliar a repetitividade (precisão) dos resultados emitidos pelos laboratórios participantes do EP/INCQS AG4 no ensaio de determinação do teor de tiram em polpa de banana, foram calculados os coeficientes de variação $(\mathrm{CV})$ dos resultados obtidos por cada laboratório para as amostras 1, 2 e 3 (polpas de banana orgânica fortificadas com a mesma solução de tiram), segundo a fórmula ${ }^{7}$ :

$\mathrm{CV}=\frac{\hat{\mathrm{S}}}{\frac{\mathrm{R} 1+\mathrm{R} 2+\mathrm{R} 3}{3}} \times 100$

onde: R1 é o resultado obtido para a Amostra 1; R2 é o resultado para a Amostra 2; R3 é o resultado para a Amostra 3 e $\mathrm{S}$ é o desvio padrão dos 3 resultados obtidos.

$\mathrm{O}$ CV considerado satisfatório no presente estudo é o estipulado pelo Codex Alimentarius como parâmetro aceitável para o ensaio de agrotóxicos em diferentes amostras de um dado hortifrutigranjeiro, fortificadas antes da extração e em níveis de concentração que variam de $>0,1 \mathrm{mg} / \mathrm{kg}$ e $<1 \mathrm{mg} / \mathrm{kg}$, que é $\leq 15 \%{ }^{7}$.

A avaliação do desempenho dos laboratórios foi realizada através do cálculo do Índice $Z$, representando uma medida da distância relativa do resultado obtido pelo laboratório em relação ao valor de referência do ensaio de proficiência ${ }^{15}$.

$\mathrm{O}$ valor de $\mathrm{Z}$ é calculado da seguinte forma:

$x_{i j}=\frac{\bar{x}_{i j}-\hat{X}}{\hat{S}}$

onde: $\hat{X}$ é o resultado obtido pelo participante i para a amostra j; 
Tabela 1. Resultados obtidos para teste de homogeneidade da amostra utilizada no EP/INCQS AG4

\begin{tabular}{|c|c|c|c|c|c|c|c|c|c|c|}
\hline & & & & & Amostra & & & & & \\
\hline & 1 & 2 & 3 & 4 & 5 & 6 & 7 & 8 & 9 & 10 \\
\hline Alíquota 1(mg/kg) & 0,318 & 0,293 & 0,353 & 0,320 & 0,321 & 0,314 & 0,335 & 0,300 & 0,293 & 0,349 \\
\hline Alíquota 2(mg/kg) & 0,314 & 0,299 & 0,292 & 0,340 & 0,303 & 0,321 & 0,315 & 0,283 & 0,301 & 0,312 \\
\hline
\end{tabular}

$\overline{\mathrm{X}}_{\mathrm{ij}}$ é a média dos resultados obtidos pelo laboratório de referência; $\hat{S}^{1 j}$ é o desvio padrão calculado através da Equação de Horwitz, para níveis de concentração $>120$ ppb e $<13,8 \%$, ou seja ${ }^{16}$ :

$\hat{S}=\frac{0,02 \times\left(\hat{X} \times 10^{-6}\right)^{0,8495}}{10^{-6}}$

Foi calculado um Índice $\mathrm{Z}$ para cada resultado emitido pelos laboratórios. Dessa forma, cada laboratório obteve 3 diferentes Índices $\mathrm{Z}$ relativos às Amostras 1, 2 e 3.

Com os resultados obtidos de $\mathrm{Z}_{\mathrm{ij}}$ foi aplicado o seguinte critério para avaliação do desempenho do laboratório para o ensaio em questão:

$\left|Z_{\mathrm{ij}}\right| \leq 2 \rightarrow$ Desempenho Satisfatório

$2<\left|Z_{\mathrm{ij}}\right|<3 \rightarrow$ Desempenho Questionável

$\left|Z_{\mathrm{ij}}\right| \geq 3 \rightarrow$ Desempenho Insatisfatório

\section{RESULTADOS E DISCUSSÃO}

\section{Resultado do teste de homogeneidade}

Os resultados obtidos pelo INCQS na determinação de ditiocarbamatos em polpa de banana, com fins a determinar a homogeneidade da amostra utilizada no EP/INCQS AG4, encontram-se na Tabela 1.

A Tabela 2 apresenta o resultado obtido no teste de homogeneidade utilizando a análise de variância (ANOVA) fator único. Considerando que o teste $\mathrm{F}_{(\alpha-0,05)}$ indicou o valor $\mathrm{F}_{\text {calculado }}$ de 1,24015 , bem menor que o $\mathrm{F}_{\text {críico }}$ de 3,02038 , a amostra foi considerada homogênea em relação ao agrotóxico tiram.

Tabela 2. Anova: fator único para o agrotóxico tiram

\begin{tabular}{lcccccc}
\hline $\begin{array}{l}\text { Fonte } \\
\text { da variação }\end{array}$ & SQ & gl & MQ & F & valor-P & F crítico \\
\hline Amostras & 0,0037 & 9 & 0,00041 & 1,24015 & 0,36900 & 3,02038 \\
Repetitividade & 0,0033 & 10 & 0,00033 & & & \\
Total & 0,0071 & 19 & & & & \\
\hline
\end{tabular}

\section{Resultado do teste de estabilidade}

Os resultados obtidos pelo INCQS na análise de tiram em polpa de banana, na determinação da estabilidade da amostra utilizada no EP/INCQS AG4, são apresentados na Figura 1.

A Tabela 3 apresenta os resultados obtidos na estimativa da variância dos valores utilizados na regressão linear. Considerando que o coeficiente angular da reta obtida na análise de regressão (Variável $\mathrm{X}_{1}$ na Tabela 3 ) foi aproximadamente zero para o agrotóxico estudado, a amostra foi considerada estável em relação a este.

\section{Resultados do laboratório de referência}

O laboratório VWA/KvW utilizou duas metodologias distintas

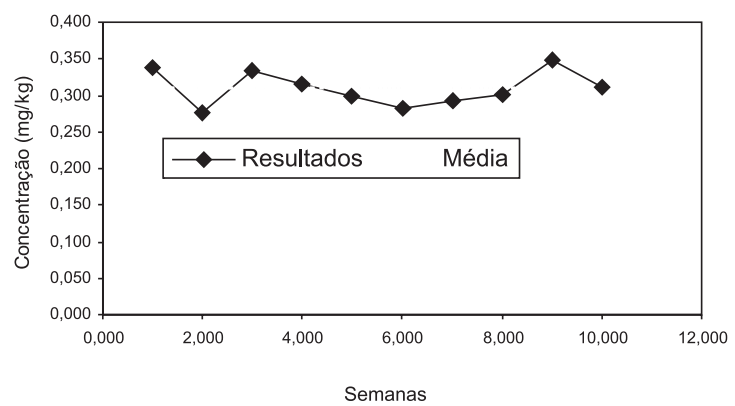

Figura 1. Gráfico de estabilidade do agrotóxico tiram em polpa de banana durante 10 semanas

Tabela 3. Análise de regressão para o agrotóxico tiram

\begin{tabular}{lccc}
\hline & $\mathrm{gl}$ & $\mathrm{SQ}$ & $\mathrm{MQ}$ \\
\hline Regressão & 1 & $1,55827 \mathrm{E}-06$ & $1,55827 \mathrm{E}-06$ \\
Resíduo & 6 & 0,001416 & 0,000236 \\
Total & 7 & 0,001418 & - \\
\hline & Coeficientes & Erro padrão & Stat t \\
\hline Interseção & 0,312909 & 0,008525 & 36,705608 \\
Variável $\mathrm{X}_{1}$ & $-3,44368 \mathrm{E}-05$ & 0,000424 & $-0,081245$ \\
\hline
\end{tabular}

na análise de ditiocarbamatos presentes nas 3 amostras enviadas a ele: cromatografia em fase gasosa com detecção por espectrometria em massa (CG-EM), metodologia 1, e cromatografia em fase gasosa com detecção por captura de elétrons (CG-DCE), metodologia $2^{12}$. Portanto, o laboratório de referência enviou 6 resultados: 3 para cada metodologia.

As médias dos resultados obtidos foram $0,250 \pm 0,009 \mathrm{mg} / \mathrm{kg}$ pela metodologia 1 e $0,250 \pm 0,008 \mathrm{mg} / \mathrm{kg}$ pela metodologia 2 .

\section{Resultados dos participantes e discussões}

A obtenção de amostras artificialmente contaminadas para o ensaio de proficiência de ditiocarbamatos encontrou algumas dificuldades: pequena estabilidade dos ditiocarbamatos quando em mistura com polpas de frutas e legumes, levando a sua rápida degradação, possivelmente por ação enzimática ${ }^{17}$; baixa solubilidade destas substâncias em água e na maioria de solventes orgânicos, característica esta que gerou publicações somente para estudos interlaboratoriais em matrizes como uvas secas e produtos inertes como lactose e amido ${ }^{10,18}$. Por outro lado, a introdução de ditiocarbamatos a materiais inertes sólidos pode gerar uma variável nova, referente à homogeneização entre sólidos.

A alternativa encontrada para realização do EP, foi o envio da solução do tiram e da matriz orgânica do hortifrutigranjeiro separadamente, para que o laboratório participante fizesse a mistura desses no momento da determinação analítica evitando, assim, sua degradação.

Uma vez que as bananas utilizadas no presente estudo eram advindas de plantação orgânica, todo o tiram determinado nas amostras supunha-se ser proveniente da solução de fortificação adicionada. 
Como foi estabelecido que fossem utilizadas $200 \mathrm{~g}$ da amostra de polpa de banana e a esta fosse adicionado todo o conteúdo da solução de tiram, o valor teórico dessa substância adicionado às amostras foi igual a $0,273 \mathrm{mg} / \mathrm{kg}$, que se refere à concentração do tiram adicionada a banana sem a determinação analítica.

Considerando-se que o valor médio das concentrações de tiram em polpa de banana obtidos pelo laboratório de referência foi de 0,250 e o valor teórico de $0,273 \mathrm{mg} / \mathrm{kg}$, concluímos estarem os resultados compatíveis, uma vez que apresentam desvios inferiores a $15 \%$, conforme recomendado pelo organismo internacional Codex Alimentarius $^{7}$.

Os resultados obtidos pelos participantes do EP/INCQS AG4 e seus respectivos índices $\mathrm{Z}$ e CVs podem ser observados na Tabela 4. Os resultados considerados questionáveis ou insatisfatórios, segundo o critério adotado no presente estudo, encontram-se em itálico.

Tabela 4. Índices $\mathrm{Z}$ e Coeficientes de Variação (CV) obtidos pelos laboratórios participantes do EP/INCQS AG4 na análise das amostras 1,2 e 3

Código do Am 1 Índice Am 2 Índice $\mathrm{Am} 3$ Índice $\mathrm{CV}$ Laboratório mg/kg Z Am $1 \mathrm{mg} / \mathrm{kg} \mathrm{Z}$ Am 2 mg/kg Z ${ }_{3} \mathrm{Am} 3$

\begin{tabular}{lccccccc}
\hline 001 & 0,41 & 3,16 & 0,39 & 2,76 & 0,25 & $-0,04$ & 24,9 \\
002 & 0,37 & 2,28 & 0,24 & $-0,24$ & 0,35 & 1,87 & 21,3 \\
003 & 0,33 & 1,56 & 0,32 & 1,36 & 0,33 & 1,56 & 1,8 \\
004 & 0,19 & $-1,24$ & 0,17 & $-1,64$ & 0,12 & $-2,64$ & 22,5 \\
005 & 0,31 & 1,16 & 0,31 & 1,16 & 0,30 & 0,96 & 1,9 \\
006 & 0,0 & $-5,04$ & 0,28 & 0,56 & 0,29 & 0,76 & 86,6 \\
007 & 0,23 & $-0,44$ & 0,33 & 1,56 & 0,17 & $-1,64$ & 33,2 \\
008 & 0,0 & $-5,04$ & 0,33 & 1,56 & 0,20 & $-1,04$ & 94,1 \\
009 & 0,29 & 0,76 & 0,30 & 0,96 & 0,29 & 0,76 & 2,0 \\
010 & 0,29 & 0,76 & 0,35 & 1,96 & 0,28 & 0,56 & 12,3 \\
011 & 0,12 & $-2,62$ & 0,16 & $-1,94$ & 0,16 & $-1,88$ & 14,2 \\
012 & 0,22 & $-0,56$ & 0,22 & $-0,58$ & 0,22 & $-0,56$ & 0,3 \\
013 & 0,21 & $-0,84$ & 0,18 & $-1,44$ & 0,20 & $-1,04$ & 7,8 \\
014 & 0,29 & 0,76 & 0,29 & 0,76 & 0,28 & 0,56 & 2,0 \\
\hline
\end{tabular}

Nota: Para os laboratórios que expressaram seus resultados como $\leq$ ao valor mínimo de detecção do equipamento ou ND (não detectado), considerou-se o valor obtido como zero.

Para melhor visualização do desempenho de cada participante, foi construído um gráfico dos Índices $\mathrm{Z}_{\mathrm{i} j}$ obtidos, o que pode ser observado na Figura 2.

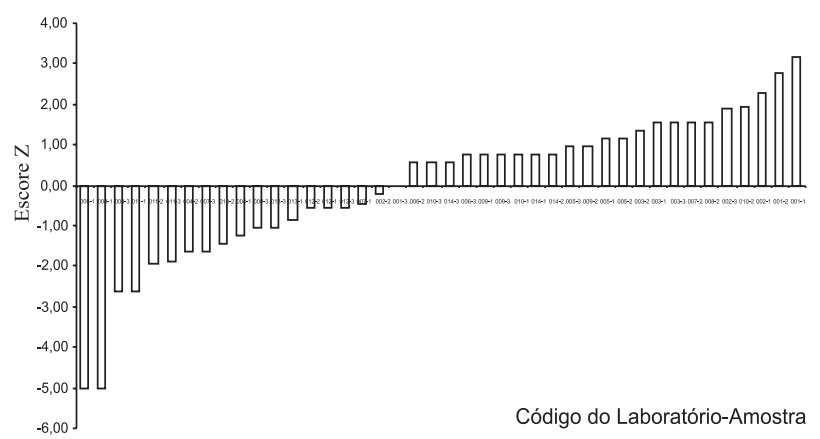

Figura 2. Índices $Z_{i j}$ obtidos pelos laboratórios participantes do EP/INCQS AG4 - determinação do teor de tiram em polpa de banana - para cada amostra analisada
Na Tabela 4 e Figura 2 observa-se que dentre os 14 laboratórios participantes do EP, 8 (50\%) obtiveram tanto o coeficiente de variação como os 3 Índices $\mathrm{Z}$ analisados satisfatórios.

\section{CONCLUSÕES}

A proposta do ensaio de proficiência organizado pelo INCQS foi motivar os laboratórios participantes para que realizem sua própria avaliação. É esperado que no caso de um resultado insatisfatório, o laboratório abra uma não-conformidade, verifique as possíveis causas que levaram a esta e estipulem ações corretivas, com prazos e responsáveis para sua implementação.

É importante lembrar que existem limitações quanto ao uso de resultados em ensaios de proficiência para a determinação de competência de um laboratório. Um desempenho mal sucedido, por ex., pode representar um desvio aleatório de um estado de competência contínuo, sendo também importante avaliar o desempenho histórico do laboratório, além de avaliações in loco, para uma adequada verificação da confiabilidade dos resultados por ele emitidos.

Podemos considerar que os resultados obtidos pelos laboratórios participantes do EP para determinação de tiram em polpa de banana podem melhorar principalmente quanto ao aspecto dos altos índices de CV e de resultados falso-negativos.

A participação do laboratório em EP é um indicativo da preocupação com a qualidade de seus dados e já é um grande passo para o aumento da sua confiabilidade.

\section{REFERÊNCIAS}

1. http://www.anvisa.gov.br/toxicologia/monografias/index.htm, acessada em Janeiro 2006.

2. WHO (World Health Organization); Pesticides Residues in Food 1993. Evaluations. Part II Toxicology, International Programme on Chemical Safety: Geneva, 1994.

3. Vettorazzi, G.; Almeida, W. F.; Burin, G. J.; Jaeger, R. B.; Puga, F. R.; Rhade, A. F.; Reyes, F. G.; Chvartsman, S.; Teratogenesis, Carcinogenesis, and Mutagenesis 1995, 15, 313.

4. http://www.anvisa.gov.br/toxicologia/resíduos/rel_anual2002_an2.pdf, acessada em Janeiro 2006.

5. Caldas, E. D.; Miranda, M. C. C.; Conceição, M. H.; de Souza, L. C. K. R.; Food Chem. Toxicol. 2004, 42, 1877.

6. Associação Brasileira de Normas Técnicas, NBR/ISO/IEC 17025; Requisitos Técnicos para a Competência Técnica de Laboratório de Ensaios e Calibração, Rio de Janeiro, 2005.

7. Codex Alimentarius Commission; Pesticide Residues in Food. Methods of analysis and sampling, $2^{\text {nd }}$ ed., part 1, Roma 2000, vol. 2A, part 1.

8. Thompson, M.; Ellison, S. L. R.; Wood, R.; Pure Appl. Chem. 2006, 78, 145.

9. Chui, Q. S. H.; Iamashita, C. O.; Bispo, J. M. A.; Quim. Nova 2005, 28, 497.

10. Keppel, G. E.; J. AOAC 1971, 54, 528.

11. Cullen, T. E.; Anal. Chem. 1964, 36, 221.

12. de Kok, A.; van Bodegraven, P.; Resumos do $4^{\text {th }}$ European Pesticide Residues Workshop, Rome, Italy, 2002.

13. Vryas, Z.; Papadakis, E. N.; Mourkidou, E. P.; J. Agric. Food Chem. 2002, $50,2220$.

14. Rohers, G.; Weckert, C.; da Silva, R. C.; Zanella, R.; Pizzutti, I. R.; de Kok, A.; Resumos do $13^{\circ}$ Encontro Nacional de Química Analítica, Niterói, Brasil, 2006.

15. Thompson, M.; Analyst 2000, 125, 385.

16. Associação Brasileira de Normas Técnicas, NBR/ISO/IEC GUIA 43; Ensaios de Proficiência por Comparações Interlaboratoriais. Parte 1: Desenvolvimento e Operação de Programas de Ensaios de Proficiência, Rio de Janeiro, 1999.

17. Vuik, J.; van Dinter, R.; de Vos, R. H.; J. Agric. Food Chem. 1992, 40, 604.

18. Gilbasch, W.; Resumos do $4^{\text {th }}$ European Pesticides Residues Workshop, Rome, Italy, 2002. 